\title{
FDM for Elliptic Equations with Bitsadze-Samarskii-Dirichlet Conditions
}

\author{
Allaberen Ashyralyev ${ }^{1,2}$ and Fatma Songul Ozesenli Tetikoglu ${ }^{1}$ \\ ${ }^{1}$ Department of Mathematics, Fatih University, 34500 Istanbul, Turkey \\ ${ }^{2}$ Department of Mathematics, ITTU, 74400 Ashgabat, Turkmenistan
}

Correspondence should be addressed to Fatma Songul Ozesenli Tetikoglu, ftetikoglu@fatih.edu.tr

Received 8 April 2012; Accepted 6 May 2012

Academic Editor: Ravshan Ashurov

Copyright (C) 2012 A. Ashyralyev and F. S. Ozesenli Tetikoglu. This is an open access article distributed under the Creative Commons Attribution License, which permits unrestricted use, distribution, and reproduction in any medium, provided the original work is properly cited.

A numerical method is proposed for solving nonlocal boundary value problem for the multidimensional elliptic partial differential equation with the Bitsadze-Samarskii-Dirichlet condition. The first and second-orders of accuracy stable difference schemes for the approximate solution of this nonlocal boundary value problem are presented. The stability estimates, coercivity, and almost coercivity inequalities for solution of these schemes are established. The theoretical statements for the solutions of these nonlocal elliptic problems are supported by results of numerical examples.

\section{Introduction}

Many problems in fluid mechanics, dynamics, elasticity, and other areas of engineering, physics, and biological systems lead to partial differential equations of elliptic type. The role played by coercive inequalities in the study of local boundary-value problems for elliptic and parabolic differential equations is well known (see, e.g., $[1,2]$ ).

In the present paper, we consider the Bitsadze-Samarskii type nonlocal boundary value problem

$$
\begin{gathered}
-\frac{d^{2} u(t)}{d t^{2}}+A u(t)=f(t), \quad(0<t<1), \\
u^{\prime}(0)=\varphi, \\
u^{\prime}(1)=\beta u^{\prime}(\lambda)+\psi, \quad|\beta| \leq 1,0 \leq \lambda<1
\end{gathered}
$$


for elliptic differential equations in a Hilbert space $H$ with self-adjoint positive definite operator $A$. It is known (see, e.g., [3-11]) that various nonlocal boundary value problems for elliptic equations can be reduced to the boundary value problem (1.1). The simply nonlocal boundary value problem was presented and investigated for the first time by Bitsadze and Samarskii [12]. Further, methods of solutions of Bitsadze-Samarskii nonlocal boundary value problems for elliptic differential equations have been studied extensively by many researchers (see [13-21] and the references given therein). satisfied.

A function $u(t)$ is called a solution of problem (1.1) if the following conditions are

(i) $u(t)$ is twice continuously differentiable on the segment $[0,1]$. Derivatives at the endpoints of the segment are understood as the appropriate unilaterial derivatives.

(ii) The element $u(t)$ belongs to $D(A)$ for all $t \in[0,1]$, and the function $A u(t)$ is continuous on $[0,1]$.

(iii) $u(t)$ satisfies the equation and nonlocal boundary condition in (1.1).

Let $\Omega$ be the open unit cube in $R^{n}\left(x=\left(x_{1}, \ldots, x_{n}\right): 0<x_{k}<1,1 \leq k \leq n\right)$ with boundary $S, \bar{\Omega}=\Omega \cup S$. In present paper, we are interested in studying the stable difference schemes for the numerical solution of the following nonlocal boundary value problem for the multidimensional elliptic equation

$$
\begin{gathered}
-u_{t t}-\sum_{r=1}^{n}\left(a_{r}(x) u_{x_{r}}\right)_{x_{r}}+\delta u=f(t, x), \quad 0<t<1, \\
x=\left(x_{1}, \ldots, x_{n}\right) \in \Omega, \\
u_{t}(0, x)=\varphi(x), \\
u_{t}(1, x)=\beta u_{t}(\lambda, x)+\psi(x), \quad x \in \bar{\Omega},|\beta| \leq 1,0 \leq \lambda<1, \\
u(t, x)=0, \quad 0 \leq t \leq 1, \quad x \in S, S=\partial \bar{\Omega} .
\end{gathered}
$$

Here $\psi(x), \varphi(x)(x \in \bar{\Omega})$, and $f(t, x)(t \in(0,1), x \in \Omega)$ are given smooth functions, $\delta$ is a large positive constant and $a_{r}(x) \geq a>0$.

In the present paper, the first and second-orders of accuracy difference schemes are presented for the approximate solution of problem (1.2). The stability and coercive stability estimates for the solution of these difference schemes are obtained. A numerical method is proposed for solving nonlocal boundary value problem for the multidimensional elliptic partial differential equation with the Bitsadze-Samarskii-Dirichlet condition. A procedure of modified Gauss elimination method is used for solving these difference schemes in the case of two-dimensional elliptic partial differential equations. 


\section{Difference Schemes: Well-Posedness}

The discretization of problem (1.2) is carried out in two steps. In the first step let us define the grid sets as follows:

$$
\begin{gathered}
\widetilde{\Omega}_{h}=\left\{x: x=x_{m}=\left(h_{1} m_{1}, \ldots, h_{n} m_{n}\right), m=\left(m_{1}, \ldots, m_{n}\right),\right. \\
\left.0 \leq m_{r} \leq N_{r}, h_{r} N_{r}=L, r=1, \ldots, n\right\} \\
\Omega_{h}=\widetilde{\Omega}_{h} \cap \Omega, \quad S_{h}=\widetilde{\Omega}_{h} \cap S .
\end{gathered}
$$

We introduce the Hilbert space $L_{2 h}=L_{2}\left(\tilde{\Omega}_{h}\right)$ of the grid functions $\varphi^{h}(x)=\left\{\varphi\left(h_{1} m_{1}, \ldots\right.\right.$, $\left.h_{n} m_{n}\right)$ \} defined on $\widetilde{\Omega}_{h}$, equipped with the norm

$$
\left\|\varphi^{h}\right\|_{L_{2}\left(\tilde{\Omega}_{h}\right)}=\left(\sum_{x \in \overline{\Omega_{h}}}\left|\varphi^{h}(x)\right|^{2} h_{1} \cdots h_{n}\right)^{1 / 2}
$$

and the Hilbert space $W_{2}^{2}\left(\widetilde{\Omega}_{h}\right)$ defined on $\widetilde{\Omega}_{h}$, equipped with the norm

$$
\begin{aligned}
\left\|\varphi^{h}\right\|_{W_{2}^{2}\left(\tilde{\Omega}_{h}\right)}= & \left(\sum_{x \in \tilde{\Omega}_{h}}\left|\varphi^{h}(x)\right|^{2} h_{1} \cdots h_{n}\right)^{1 / 2} \\
& +\left(\sum_{x \in \tilde{\Omega}_{h}} \sum_{r=1}^{n}\left|\varphi_{x_{r}, m_{r}}^{h}\right|^{2} h_{1} \cdots h_{n}\right)^{1 / 2}+\left(\sum_{x \in \tilde{\Omega}_{h}} \sum_{r=1}^{n}\left|\varphi_{x_{r} \bar{x}_{r}, m_{r}}^{h}\right|^{2} h_{1} \cdots h_{n}\right)^{1 / 2} .
\end{aligned}
$$

Finally, we introduce the Banach spaces $C\left([0,1]_{\tau}, L_{2 h}\right)$ and $C^{\alpha}\left([0,1]_{\tau}, L_{2 h}\right)$ of grid abstract function $\left\{\varphi_{k}^{h}(x)\right\}_{1}^{N-1}$ defined on $[0,1]_{\tau}$ with values $L_{2 h}$, equipped with the following norms:

$$
\begin{gathered}
\left\|\left\{\varphi_{k}^{h}\right\}_{1}^{N-1}\right\|_{C\left([0,1]_{\tau}, L_{2 h}\right)}=\max _{1 \leq k \leq N-1}\left\|\varphi_{k}^{h}\right\|_{L_{2 h}{ }^{\prime}} \\
\left\|\left\{\varphi_{k}^{h}\right\}_{1}^{N-1}\right\|_{C^{\alpha}\left([0,1]_{\tau}, L_{2 h}\right)}=\max _{1 \leq k \leq N-1}\left\|\varphi_{k}^{h}\right\|_{L_{2 h}}+\sup _{1 \leq k<k+r \leq N-1} \frac{\left\|\varphi_{k+r}-\varphi_{k}\right\|_{L_{2 h}}}{(r \tau)^{\alpha}} .
\end{gathered}
$$

To the differential operator $A$ generated by the problem (1.2) we assign the difference operator $A_{h}^{x}$ by the formula

$$
A_{h}^{x} u^{h}=-\sum_{r=1}^{n}\left(a_{r}(x) u_{\bar{x}_{r}}^{h}\right)_{x_{r}, m_{r}}+\delta u_{x_{r}}^{h}
$$

acting in the space of grid functions $u^{h}(x)$, satisfying the condition $u^{h}(x)=0$ for all $x \in S_{h}$. It is known that $A_{h}^{x}$ is a self-adjoint positive definite operator in $L_{2}\left(\widetilde{\Omega}_{h}\right)$. With the help of 
$A_{h}^{x}$, we arrive at the nonlocal boundary value problem for an infinite system of the following ordinary differential equations:

$$
\begin{gathered}
-\frac{d^{2} u^{h}(t, x)}{d t^{2}}+A_{h}^{x} u^{h}(t, x)=f^{h}(t, x), \quad 0<t<1, \quad x \in \Omega_{h}, \\
u_{t}^{h}(0, x)=\varphi^{h}(x), \quad u_{t}^{h}(1, x)=\beta u_{t}^{h}(\lambda, x)+\psi^{h}(x), \quad x \in \tilde{\Omega}_{h} .
\end{gathered}
$$

In the second step, we replaced problem (2.6) by the first-order of accuracy difference scheme as follows:

$$
\begin{gathered}
-\frac{u_{k+1}^{h}(x)-2 u_{k}^{h}(x)+u_{k-1}^{h}(x)}{\tau^{2}}+A_{h}^{x} u_{k}^{h}(x)=f_{k}^{h}(x), \\
f_{k}^{h}(x)=f^{h}\left(t_{k}, x\right), \quad t_{k}=k \tau, 1 \leq k \leq N-1, N \tau=1, x \in \Omega_{h}, \\
\frac{u_{1}^{h}(x)-u_{0}^{h}(x)}{\tau}=\varphi^{h}(x), \quad x \in \widetilde{\Omega}_{h}, \\
\frac{u_{N}^{h}(x)-u_{N-1}^{h}(x)}{\tau}=\beta \frac{u_{[\lambda / \tau]+1}^{h}(x)-u_{[\lambda / \tau]}^{h}(x)}{\tau}+\psi^{h}(x), \quad x \in \tilde{\Omega}_{h}
\end{gathered}
$$

and the second order of accuracy difference scheme as follows:

$$
\begin{gathered}
-\frac{u_{k+1}^{h}(x)-2 u_{k}^{h}(x)+u_{k-1}^{h}(x)}{\tau^{2}}+A_{h}^{x} u_{k}^{h}(x)=f_{k}^{h}(x), \\
f_{k}^{h}(x)=f^{h}\left(t_{k}, x\right), \quad t_{k}=k \tau, 1 \leq k \leq N-1, N \tau=1, \quad x \in \Omega_{h}, \\
\frac{-3 u_{0}^{h}(x)+4 u_{1}^{h}(x)-u_{2}^{h}(x)}{2 \tau}=\varphi^{h}(x), \quad x \in \tilde{\Omega}_{h,} \\
\frac{u_{N-2}^{h}(x)-4 u_{N-1}^{h}(x)+3 u_{N}^{h}(x)}{2 \tau}=\beta\left[\frac{u_{[\lambda / \tau]-1}^{h}(x)-4 u_{[\lambda / \tau]}^{h}(x)+3 u_{[\lambda / \tau]+1}^{h}(x)}{2 \tau}\right. \\
\left.+\frac{u_{[\lambda / \tau]+1}^{h}(x)-2 u_{[\lambda / \tau]}^{h}(x)+u_{[\lambda / \tau]-1}^{h}(x)}{\tau}\left(\frac{\lambda}{\tau}-\left[\frac{\lambda}{\tau}\right]\right)\right] \\
+\psi^{h}(x), \quad x \in \tilde{\Omega}_{h} .
\end{gathered}
$$

Now, we will study the well-posedness of (2.7) and (2.8). We have the following theorem on stability of (2.7) and (2.8). 
Theorem 2.1. Let $\tau$ and $|h|$ be sufficiently small positive numbers. Then the solutions of difference schemes (2.7) and (2.8) satisfy the following stability estimate:

$$
\max _{1 \leq k \leq N}\left\|u_{k}^{h}\right\|_{L_{2 h}} \leq M_{1}\left[\left\|\varphi^{h}\right\|_{L_{2 h}}+\left\|\psi^{h}\right\|_{L_{2 h}}+\max _{1 \leq k \leq N-1}\left\|f_{k}^{h}\right\|_{L_{2 h}}\right]
$$

where $M_{1}$ does not depend on $\tau, h, \psi^{h}(x), \varphi^{h}(x)$ and $f_{k}^{h}(x), 1 \leq k \leq N-1$.

Proof. The proof of (2.9) is based on the following formula:

$$
\begin{aligned}
& u_{k}^{h}(x)=\left(I-R^{2 N}\right)^{-1}\{(\left(R^{k}-R^{2 N-k}\right) u_{0}^{h}(x)+\left(R^{N-k}-R^{N+k}\right) u_{N}^{h}(x) \\
&\left.-\left(R^{N-k}-R^{N+k}\right)\left(2 I+\tau B_{h}^{x}\right)^{-1}\left(B_{h}^{x}\right)^{-1} \sum_{i=1}^{N-1}\left(R^{N-1-i}-R^{N-1+i}\right) f_{i}^{h}(x) \tau\right\} \\
&+\left(2 I+\tau B_{h}^{x}\right)^{-1}\left(B_{h}^{x}\right)^{-1} \sum_{i=1}^{N-1}\left(R^{|k-i|-1}-R^{k+i-1}\right) f_{i}^{h}(x) \tau \quad \text { for } k=1, \ldots, N-1,
\end{aligned}
$$

where

$$
\begin{aligned}
u_{0}^{h}(x)=P_{\tau}\left(I+\tau B_{h}^{x}\right)\left(2 I+\tau B_{h}^{x}\right)^{-1}\left(B_{h}^{x}\right)^{-1} \\
\times\left[(I+R) R^{N-2} \sum_{i=1}^{N-1}\left(R^{N-i}-R^{N+i}\right) f_{i}^{h}(x) \tau\right. \\
\quad+(I+R)\left[I+R^{2 N-2}-\beta\left(R^{N-[\lambda / \tau]-1}+R^{N+[\lambda / \tau]}\right)\right] \\
\quad \times \sum_{i=1}^{N-1} R^{i-1} f_{i}^{h}(x) \tau-\beta\left(R^{N}+R^{N-1}\right) \sum_{i=1}^{[\lambda / \tau]-1} R^{[\lambda / \tau]-i} f_{i}^{h}(x) \tau \\
+\beta\left(R^{N-2}+R^{N-1}\right) \sum_{i=[\lambda / \tau]+1}^{N-1} R^{i-[\lambda / \tau]} f_{i}^{h}(x) \tau \\
\left.+\beta\left(R^{N}+R^{N-1}\right) \sum_{i=1}^{N-1} R^{[\lambda / \tau]+i} f_{i}^{h}(x) \tau-\beta\left(R^{N-1}+R^{N}\right) f_{[\lambda / \tau]}^{h}(x) \tau\right] \\
-P_{\tau}(I-R)^{-1}\left[I+R^{2 N-1}-\beta\left(R^{N-[\lambda / \tau]-1}+R^{N+[\lambda / \tau]}\right)\right] \varphi^{h}(x) \tau \\
+P_{\tau}(I-R)^{-1}\left(R^{N-1}+R^{N}\right) \psi^{h}(x) \tau,
\end{aligned}
$$




$$
\begin{aligned}
u_{N}^{h}(x)=P_{\tau}\left(I+\tau B_{h}^{x}\right)\left(2 I+\tau B_{h}^{x}\right)^{-1}\left(B_{h}^{x}\right)^{-1} & \\
\times & {\left[\left[R^{-1}(I+R)-\beta\left(R^{N-[\lambda / \tau]-1}+R^{N+[\lambda / \tau]-1}\right)\right] \sum_{i=1}^{N-1}\left(R^{N-i}-R^{N+i}\right) f_{i}^{h}(x) \tau\right.} \\
& \quad \beta\left(I+R^{2 N-1}\right) \sum_{i=1}^{[\lambda / \tau]-1} R^{[\lambda / \tau]-i} f_{i}^{h}(x) \tau+\beta\left(I+R^{2 N-1}\right) R^{-1} \sum_{i=[\lambda / \tau]+1}^{N-1} R^{i-[\lambda / \tau]} f_{i}^{h}(x) \tau \\
& +\beta\left(I+R^{2 N-1}\right) \sum_{i=1}^{N-1} R^{[\lambda / \tau]+i} f_{i}^{h}(x) \tau-\beta\left(I+R^{2 N-1}\right) f_{[\lambda / \tau]}^{h}(x) \tau \\
& \left.+(I+R)\left[R^{N}+R^{N-1}-\beta\left(R^{[\lambda / \tau]}+R^{2 N-[\lambda / \tau]-1}\right)\right] \sum_{i=1}^{N-1} R^{i-1} f_{i}^{h}(x) \tau\right] \\
& -P_{\tau}(I-R)^{-1}\left[R^{N}+R^{2 N-1}-\beta\left(R^{[\lambda / \tau]}+R^{2 N-[\lambda / \tau]-1}\right)\right] \varphi^{h}(x) \tau \\
& +P_{\tau}(I-R)^{-1}\left(I+R^{2 N-1}\right) \psi^{h}(x) \tau, \\
P_{\tau}=[I- & \left.R^{2 N-2}-\beta\left(R^{N-[\lambda / \tau]-1}+R^{N+[\lambda / \tau]-1}\right)\right]^{-1},
\end{aligned}
$$

for (2.7), and

$$
\begin{aligned}
u_{0}^{h}(x)=D_{\tau}(I+ & \left.\tau B_{h}^{x}\right)\left(2 I+\tau B_{h}^{x}\right)^{-1}\left(B_{h}^{x}\right)^{-1} \\
\times & \left\{(I+R)\left(4 R-I-R^{2}\right)(I-3 R) R^{N-4}\left(I-R^{2 N}\right) \sum_{i=1}^{N-2}\left(R^{N-i}-R^{N+i}\right) f_{i}^{h}(x) \tau\right. \\
& -(I+R)\left(4 R-I-R^{2}\right)\left(I-R^{2 N}\right) \\
\times & {\left[3 I-R-R^{2 N-2}(I-3 R)\right.} \\
& -\beta\left[R^{N-[\lambda / \tau]-1}\left(I-3 R+2\left(\frac{\lambda}{\tau}-\left[\frac{\lambda}{\tau}\right]\right)(I-R)\right)\right. \\
\times & \quad \sum_{i=2}^{N-1} R^{i-2} f_{i}^{h}(x) \tau-\beta(I+R) R^{N-2}\left(4 R-I-R^{2}\right)\left(I-R^{2 N}\right) \\
\times & {\left[\left(I-3 R+2\left(\frac{\lambda}{\tau}-\left[\frac{\lambda}{\tau}\right]\right)(I-R)\right) \sum_{i=1}^{[\lambda / \tau]-1} R^{[\lambda / \tau]-i-1} f_{i}^{h}(x) \tau\right.} \\
& \quad+\left(3 I-R+2\left(\frac{\lambda}{\tau}-\left[\frac{\lambda}{\tau}\right]\right)(I-R)\right) \sum_{i=[\lambda / \tau]+2}^{N-1} R^{i-[\lambda / \tau]-1} f_{i}^{h}(x) \tau \\
& \left.\quad\left(I-3 R+2\left(\frac{\lambda}{\tau}-\left[\frac{\lambda}{\tau}\right]\right)(I-R)\right) \sum_{i=1}^{N-1} R^{[\lambda / \tau]+i-1} f_{i}^{h}(x) \tau\right]
\end{aligned}
$$


Abstract and Applied Analysis

$$
\begin{aligned}
&- \beta\left(I-R^{2 N}\right)(I+R) R^{N-2}\left(4 R-R^{2}-I\right)\left(4+4\left(\frac{\lambda}{\tau}-\left[\frac{\lambda}{\tau}\right]\right)\right) \\
& \times\left(f_{[\lambda / \tau]+1}^{h}(x) \tau-f_{[\lambda / \tau]}^{h}(x) \tau\right)-(I+R)(4 I-R)\left(I-R^{2 N}\right) \\
& \times {\left[3 I-R-R^{2 N-2}(I-3 R)\right.} \\
&-\beta\left(R^{N-[\lambda / \tau]-1}\left(3 I-R+2\left(\frac{\lambda}{\tau}-\left[\frac{\lambda}{\tau}\right]\right)(I-R)\right)\right. \\
&\left.\left.\quad-R^{N+[\lambda / \tau]-1}\left(I-3 R+2\left(\frac{\lambda}{\tau}-\left[\frac{\lambda}{\tau}\right]\right)(I-R)\right)\right)\right] \\
& \times f_{1}^{h}(x) \tau-(I+R) R^{N-1}\left(4 R-R^{2}-I\right)\left(I-R^{2 N}\right)\left(4 I+R^{2 N-3}(I-3 R)\right) \\
&-D_{\tau}\left(I-R^{2 N}\right)(I-R)^{-1}(I+R) R^{N-2}\left(4 R-I-R^{2}\right) 2 \tau \psi^{h}(x) \\
&+D_{\tau}\left(I-R^{2 N}\right)(I-R)^{-1} \\
& \times\left[3 I-R-R^{2 N-2}(I-3 R)\right. \\
&-\beta\left[R^{N-[\lambda / \tau]-1}\left(3 I-R+2\left(\frac{\lambda}{\tau}-\left[\frac{\lambda}{\tau}\right]\right)(I-R)\right)\right. \\
&\left.\left.-R^{N+[\lambda / \tau]-1}\left(I-3 R+2\left(\frac{\lambda}{\tau}-\left[\frac{\lambda}{\tau}\right]\right)(I-R)\right)\right]\right] 2 \tau \varphi^{h}(x),
\end{aligned}
$$$$
u_{N}^{h}(x)=D_{\tau}\left(I+\tau B_{h}^{x}\right)\left(2 I+\tau B_{h}^{x}\right)^{-1}\left(B_{h}^{x}\right)^{-1}
$$$$
\times\left\{( I - R ^ { 2 N } ) \left[(I+R)\left(4 R-I-R^{2}\right) R^{-2}(R-3 I)\right.\right.
$$$$
+\beta\left[(R-3 I)\left(3 I-R+2\left(\frac{\lambda}{\tau}-\left[\frac{\lambda}{\tau}\right]\right)(I-R)\right) R^{N-[\lambda / \tau]-1}\right.
$$$$
\left.\left.-(3 R-I)\left(I-3 R+2\left(\frac{\lambda}{\tau}-\left[\frac{\lambda}{\tau}\right]\right)(I-R)\right) R^{N+[\lambda / \tau]-1}\right]\right]
$$$$
\times \sum_{i=1}^{N-2}\left(R^{N-i}-R^{N+i}\right) f_{i}^{h}(x) \tau+(I+R)\left(I-R^{2 N}\right)\left(4 R-I-R^{2}\right)
$$$$
\times\left[(I+R) R^{N-2}\left(4 R-I-R^{2}\right)\right.
$$$$
-\beta\left[R^{[\lambda / \tau]-1}\left(I-3 R+2\left(\frac{\lambda}{\tau}-\left[\frac{\lambda}{\tau}\right]\right)(I-R)\right)\right.
$$$$
\left.\left.-R^{2 N-[\lambda / \tau]-1}\left(3 I-R+2\left(\frac{\lambda}{\tau}-\left[\frac{\lambda}{\tau}\right]\right)(I-R)\right)\right]\right] \sum_{i=2}^{N-1} R^{i-2} f_{i}^{h}(x) \tau
$$ 


$$
\begin{aligned}
& +\beta\left(I-R^{2 N}\right)\left(R-3 I+R^{2 N-2}(I-3 R)\right) \\
& \times\left[\left(I-3 R+2\left(\frac{\lambda}{\tau}-\left[\frac{\lambda}{\tau}\right]\right)(I-R)\right) \sum_{i=1}^{[\lambda / \tau]-1} R^{[\lambda / \tau]-i-1} f_{i}^{h}(x) \tau\right. \\
& +\left(3 I-R+2\left(\frac{\lambda}{\tau}-\left[\frac{\lambda}{\tau}\right]\right)(I-R)\right) \sum_{i=[\lambda / \tau]+2}^{N-1} R^{i-[\lambda / \tau]-1} f_{i}^{h}(x) \tau \\
& \left.-\left(I-3 R+2\left(\frac{\lambda}{\tau}-\left[\frac{\lambda}{\tau}\right]\right)(I-R)\right) \sum_{i=1}^{N-1} R^{[\lambda / \tau]+i-1} f_{i}^{h}(x) \tau\right] \\
& +\beta\left(R-3 I+R^{2 N-2}(3 I-R)\right)\left(I-R^{2 N}\right) \\
& \times\left(4+4\left(\frac{\lambda}{\tau}-\left[\frac{\lambda}{\tau}\right]\right)(I-R)\right)\left(f_{[\lambda / \tau]+1}^{h}(x) \tau-f_{[\lambda / \tau]}^{h}(x) \tau\right) \\
& +(I+R)\left(I-R^{2 N}\right) \\
& \times\left[(I+R) R^{N-2}\left(I-4 R+R^{2}\right)\right. \\
& -\beta\left[R^{[\lambda / \tau]-1}\left(I-3 R+2\left(\frac{\lambda}{\tau}-\left[\frac{\lambda}{\tau}\right]\right)(I-R)\right)\right. \\
& \left.\left.-R^{2 N-[\lambda / \tau]-1}\left(3 I-R+2\left(\frac{\lambda}{\tau}-\left[\frac{\lambda}{\tau}\right]\right)(I-R)\right)\right]\right] f_{1}^{h}(x) \tau \\
& +R\left(I-R^{2 N-1}\right)\left[R-4 I+R^{2 N-4}\left(I+R^{2}-3 R\right)\left(3 I-R-R^{2}(4 I-R)\right)\right. \\
& -\beta\left[R^{N-[\lambda / \tau]-1}\left(3 I-R+2\left(\frac{\lambda}{\tau}-\left[\frac{\lambda}{\tau}\right]\right)(I-R)\right)\right. \\
& \times\left(3 R-I+R^{2 N}(3 I-R)\right) \\
& -R^{N+[\lambda / \tau]-1}\left(I-3 R+2\left(\frac{\lambda}{\tau}-\left[\frac{\lambda}{\tau}\right]\right)(I-R)\right) \\
& \left.\left.\left.\times\left(3 I-R+R^{2 N}(I-3 R)\right)\right]\right] f_{N-1}^{h}(x) \tau\right\} \\
& +D_{\tau}\left(I-R^{2 N}\right)(I-R)^{-1}\left(R-3 I+R^{2 N}(I-3 R)\right) 2 \tau \varphi^{h}(x) \\
& -D_{\tau}\left(I-R^{2 N}\right)(I-R)^{-1} \\
& \times\left[R^{N-2}(I+R)\left(R^{2}-4 R+I\right)\right. \\
& -\beta\left[R^{[\lambda / \tau]-1}\left(I-3 R+2\left(\frac{\lambda}{\tau}-\left[\frac{\lambda}{\tau}\right]\right)(I-R)\right)\right. \\
& \left.\left.-R^{2 N-[\lambda / \tau]-1}\left(3 I-R+2\left(\frac{\lambda}{\tau}-\left[\frac{\lambda}{\tau}\right]\right)(I-R)\right)\right]\right] 2 \tau \psi^{h}(x),
\end{aligned}
$$




$$
\begin{gathered}
D_{\tau}=\left(I-R^{2 N}\right)^{-1} \\
\times\left\{\left[-(3 I-R)^{2}+(I-3 R)^{2}\right]\right. \\
-\beta\left[-R^{N+[\lambda / \tau]-3}(I-3 R)\left(I-3 R+2\left(\frac{\lambda}{\tau}-\left[\frac{\lambda}{\tau}\right]\right)(I-R)\right)\right. \\
\left.\left.-R^{N-[\lambda / \tau]-1}(3 I-R)\left(3 I-R+2\left(\frac{\lambda}{\tau}-\left[\frac{\lambda}{\tau}\right]\right)(I-R)\right)\right]\right\}^{-1}, \\
R=\left(I+\tau B_{h}^{x}\right)^{-1}, \\
B_{h}^{x}=\frac{1}{2}\left(\tau A_{h}^{x}+\sqrt{4 A_{h}^{x}+\tau^{2}\left(A_{h}^{x}\right)^{2}}\right)
\end{gathered}
$$

for (2.8), and the symmetry properties of the difference operator $A_{h}^{x}$ defined by the formula (2.5)

Difference schemes (2.7) and (2.8) are ill-posed in $C\left([0,1]_{\tau}, L_{2 h}\right)$. We have the following theorem on almost coercive stability.

Theorem 2.2. Let $\tau$ and $|h|$ be sufficiently small positive numbers. Then the solutions of difference schemes (2.7) and (2.8) satisfy the following almost coercive stability estimate:

$$
\begin{aligned}
& \max _{1 \leq k \leq N-1}\left\|\frac{u_{k+1}^{h}-2 u_{k}^{h}+u_{k-1}^{h}}{\tau^{2}}\right\|_{L_{2 h}}+\max _{1 \leq k \leq N-1}\left\|u_{k}^{h}\right\|_{W_{2 h}^{2}} \\
& \quad \leq M_{2}\left[\left\|\varphi^{h}\right\|_{W_{2 h}^{2}}+\left\|\psi^{h}\right\|_{W_{2 h}^{2}}+\ln \frac{1}{\tau+|h|} \max _{1 \leq k \leq N-1}\left\|f_{k}^{h}\right\|_{L_{2 h}}\right],
\end{aligned}
$$

where $M_{2}$ does not depend on $\tau, h, \psi^{h}(x), \varphi^{h}(x)$, and $f_{k}^{h}(x), 1 \leq k \leq N-1$.

Proof. The proof of (2.14) is based on the formulas (2.11), (2.12), (2.13), the symmetry properties of the difference operator $A_{h}^{x}$ defined by the formula (2.5) and on the following theorem on well-posedness of the elliptic difference problem.

Theorem 2.3. For the solutions of the elliptic difference problem

$$
\begin{gathered}
A_{h}^{x} u^{h}(x)=w^{h}(x), \quad x \in \Omega_{h}, \\
u^{h}(x)=0, \quad x \in S_{h}
\end{gathered}
$$

the following coercivity inequality holds (see [21]):

$$
\sum_{r=1}^{n}\left\|\left(u^{h}\right)_{x_{r} \overline{x_{r}}, m_{r}}\right\|_{L_{2 h}} \leq M\left\|w^{h}\right\|_{L_{2 h}{ }^{\prime}}
$$

where $M$ does not depend on $h$ and $w^{h}(x)$. 
Theorem 2.4. Let $\varphi^{h}(x)=\psi^{h}(x)=0$. Then the difference problems (2.7) and (2.8) are well-posed in Hölder spaces $C^{\alpha}\left([0,1]_{\tau}, L_{2 h}\right)$ and the following coercivity inequality holds:

$$
\begin{aligned}
& \max _{1 \leq k \leq N-1}\left\|\left\{\frac{u_{k+1}^{h}-2 u_{k}^{h}+u_{k-1}^{h}}{\tau^{2}}\right\}_{1}^{N-1}\right\|_{C^{\alpha}\left([0,1]_{\tau^{\prime}} L_{2 h}\right)}+\left\|\left\{u_{k}^{h}\right\}_{1}^{N-1}\right\|_{C^{\alpha}\left([0,1]_{\tau^{\prime}} W_{2 h}^{2}\right)} \\
& \leq \frac{M_{3}}{\alpha(1-\alpha)} \max _{1 \leq k \leq N-1}\left\|\left\{f_{k}^{h}\right\}_{1}^{N-1}\right\|_{C^{\alpha}\left([0,1]_{\tau}, L_{2 h}\right)} .
\end{aligned}
$$

Here $M_{3}$ does not depend on $\tau, h$, and $f_{k}^{h}(x), 1 \leq k \leq N-1$.

Proof. The proof of (2.17) is based on the formula

$$
\begin{aligned}
& A_{h}^{x} u_{0}^{h}(x)-f_{1}^{h}(x)=P_{\tau}\left(I+\tau B_{h}^{x}\right)\left(2 I+\tau B_{h}^{x}\right)^{-1}(I+R) \\
& \times\left[R^{N-1} \sum_{i=1}^{N-1} \tau B_{h}^{x} R^{N-i}\left(f_{i}^{h}-f_{N-1}^{h}\right)\right. \\
& -R^{N-1} \sum_{i=1}^{N-1} \tau B_{h}^{x} R^{N+i}\left(f_{i}^{h}(x)-f_{1}^{h}(x)\right) \\
& -\beta R^{N} \sum_{i=1}^{[\lambda / \tau]-1} \tau B_{h}^{x} R^{[\lambda / \tau]-i}\left(f_{i}^{h}(x)-f_{[\lambda / \tau]}^{h}(x)\right) \\
& +\left[I+R^{2 N-2}-\beta\left(R^{N-[\lambda / \tau]-1}+R^{N+[\lambda / \tau]}\right)\right] \\
& \times \sum_{i=1}^{N-1} \tau B_{h}^{x} R^{i}\left(f_{i}^{h}(x)-f_{1}^{h}(x)\right) \\
& +\beta R^{N} \sum_{i=[\lambda / \tau]+1}^{N-1} \tau B_{h}^{x} R^{i-[\lambda / \tau]}\left(f_{i}^{h}(x)-f_{[\lambda / \tau]}^{h}(x)\right) \\
& +\beta R^{N} \sum_{i=1}^{N-1} \tau B_{h}^{x} R^{[\lambda / \tau]+i}\left(f_{i}^{h}(x)-f_{1}^{h}(x)\right) \\
& +R^{N-1}\left(I-R^{N-1}\right) f_{N-1}^{h}(x) \\
& +\beta\left[R^{N+[\lambda / \tau]-1}-R^{2 N-[\lambda / \tau]-1}-\tau B_{h}^{x} R^{N}\right] f_{[\lambda / \tau]}^{h}(x) \\
& +\left[R^{2 N-2}-R^{2 N-1}+R^{2 N-2}-R^{3 N-3}+R^{3 N-2}-R^{N-1}\right. \\
& +\beta\left(R^{2 N-[\lambda / \tau]-2}+R^{2 N+[\lambda / \tau]-1}\right. \\
& \left.\left.\left.+R^{2 N+[\lambda / \tau]}-R^{N+[\lambda / \tau]-1}\right)\right] f_{1}^{h}(x)\right]
\end{aligned}
$$


Abstract and Applied Analysis

$$
\begin{aligned}
& -P_{\tau}(I-R)^{-1} \tau\left[I+R^{2 N-1}-\beta\left(R^{N-[\lambda / \tau]-1}+R^{N+[\lambda / \tau]}\right)\right] A_{h}^{x} \varphi^{h} \\
& +P_{\tau}(I-R)^{-1} \tau\left(R^{N-1}+R^{N}\right) A_{h}^{x} \psi^{h}, \\
& A_{x}^{h} u_{N}^{h}(x)-f_{N-1}^{h}(x)=P_{\tau}\left(I+\tau B_{h}^{x}\right)\left(2 I+\tau B_{h}^{x}\right)^{-1} \\
& \times\left\{\left[R(I+R)-\beta\left(R^{N-[\lambda / \tau]+1}+R^{N+[\lambda / \tau]+1}\right)\right]\right. \\
& \times \sum_{i=1}^{N-1} \tau B_{h}^{x} R^{N-i}\left(f_{i}^{h}(x)-f_{N-1}^{h}(x)\right) \\
& -\left[R(I+R)-\beta\left(R^{N-[\lambda / \tau]+1}+R^{N+[\lambda / \tau]+1}\right)\right] \\
& \times \sum_{i=1}^{N-1} \tau B_{h}^{x} R^{N+i}\left(f_{i}^{h}(x)-f_{1}^{h}(x)\right) \\
& -\beta R^{2}\left(I+R^{2 N-1}\right) \sum_{i=1}^{[\lambda / \tau]-1} \tau B_{h}^{x} R^{[\lambda / \tau]-i}\left(f_{i}^{h}(x)-f_{[\lambda / \tau]}^{h}(x)\right) \\
& +\beta R\left(I+R^{2 N-1}\right) \sum_{i=[\lambda / \tau]+1}^{N-1} \tau B_{h}^{x} R^{i-[\lambda / \tau]}\left(f_{i}^{h}(x)-f_{[\lambda / \tau]}^{h}(x)\right) \\
& +\beta R^{2}\left(I+R^{2 N-1}\right) \sum_{i=1}^{N-1} \tau B_{h}^{x} R^{[\lambda / \tau]+i}\left(f_{i}^{h}(x)-f_{1}^{h}(x)\right) \\
& -\beta\left(I+R^{2 N-1}\right) \\
& \times\left[R\left(I-R^{[\lambda / \tau]-1}\right)-\left(I-R^{N-[\lambda / \tau]-1}\right)-\tau B_{h}^{x} R\right] \\
& \times f_{[\lambda / \tau]}^{h}(x) \\
& +\left[(I+R)\left(R^{2 N-2}-R^{N}-I+R\right)\right. \\
& -\beta\left(R^{N-[\lambda / \tau]+1}\right. \\
& +R^{N+[\lambda / \tau]+1}-R^{2 N-[\lambda / \tau]} \\
& -R^{2 N+[\lambda / \tau]}-R^{N-[\lambda / \tau]-1} \\
& \left.\left.+R^{N+[\lambda / \tau]-1}-R^{N-[\lambda / \tau]}+R^{N+[\lambda / \tau]}\right)\right] \\
& \times f_{N-1}^{h}(x)+\left[(I+R)\left(R^{N}-R^{2 N-1}\right)\right. \\
& +\beta\left(R^{N+[\lambda / \tau]}+R^{N+[\lambda / \tau]+1}-R^{N+[\lambda / \tau]+2}\right. \\
& +R^{2 N+[\lambda / \tau]+1}+R^{2 N+[\lambda / \tau]+1}-R^{3 N+[\lambda / \tau]} \\
& -R^{3 N+[\lambda / \tau]+1}+R^{[\lambda / \tau]+1}-R^{2 N-[\lambda / \tau]} \\
& \left.\left.\left.+R^{3 N-[\lambda / \tau]-1}\right)\right] f_{1}^{h}(x)\right\}
\end{aligned}
$$




$$
\begin{aligned}
& +P_{\tau}(I-R)^{-1} R \tau\left(I+R^{2 N-1}\right) A_{x}^{h} \psi \\
& -P_{\tau}(I-R)^{-1} R \tau\left[R^{N-1}+R^{N}-\beta\left(R^{[\lambda / \tau]}+R^{2 N-[\lambda / \tau]-1}\right)\right] A_{x}^{h} \varphi
\end{aligned}
$$

for (2.7) and

$$
\begin{aligned}
& A_{h}^{x} u_{0}^{h}(x)-f_{1}^{h}(x)=D_{\tau}\left(I+\tau B_{h}^{x}\right)\left(2 I+\tau B_{h}^{x}\right)^{-1} \\
& \times\left\{(I+R)\left(4 R-I-R^{2}\right)(I-3 R) R^{N-3}\left(I-R^{2 N}\right)\right. \\
& \times\left[\sum_{i=1}^{N-2} B_{h}^{x} \tau R^{N-i}\left(f_{i}^{h}(x)-f_{N-1}^{h}(x)\right)\right. \\
& \left.-\sum_{i=1}^{N-2} B_{h}^{x} \tau R^{N+i}\left(f_{i}^{h}(x)-f_{1}^{h}(x)\right)\right] \\
& -(I+R)\left(4 R-I-R^{2}\right)\left(I-R^{2 N}\right) \\
& \times\left[3 I-R-R^{2 N-2}(I-3 R)\right. \\
& -\beta\left[R^{N-[\lambda / \tau]-1}\left(3 I-R+2\left(\frac{\lambda}{\tau}-\left[\frac{\lambda}{\tau}\right]\right)(I-R)\right)\right. \\
& \left.\left.-R^{N+[\lambda / \tau]-1}\left(I-3 R+2\left(\frac{\lambda}{\tau}-\left[\frac{\lambda}{\tau}\right]\right)(I-R)\right)\right]\right] \\
& \times \sum_{i=2}^{N-1} B_{h}^{x} \tau R^{i-1}\left(f_{i}^{h}(x)-f_{1}^{h}(x)\right) \\
& -\beta(I+R) R^{N-2}\left(4 R-I-R^{2}\right)\left(I-R^{2 N}\right) \\
& \times\left[\left(I-3 R+2\left(\frac{\lambda}{\tau}-\left[\frac{\lambda}{\tau}\right]\right)(I-R)\right)\right. \\
& \times \sum_{i=1}^{[\lambda / \tau]-1} B_{h}^{x} \tau R^{[\lambda / \tau]-i}\left(f_{i}^{h}(x)-f_{[\lambda / \tau]}^{h}(x)\right) \\
& +\left(3 I-R+2\left(\frac{\lambda}{\tau}-\left[\frac{\lambda}{\tau}\right]\right)(I-R)\right) \\
& \times \sum_{i=[\lambda / \tau]+2}^{N-1} B_{h}^{x} \tau R^{i-[\lambda / \tau]}\left(f_{i}^{h}(x)-f_{[\lambda / \tau]}^{h}(x)\right)
\end{aligned}
$$


Abstract and Applied Analysis

$$
\begin{aligned}
& -\left(I-3 R+2\left(\frac{\lambda}{\tau}-\left[\frac{\lambda}{\tau}\right]\right)(I-R)\right) \\
& \left.\times \sum_{i=1}^{N-1} B_{h}^{x} \tau R^{[\lambda / \tau]+i}\left(f_{i}^{h}(x)-f_{1}^{h}(x)\right)\right] \\
& -\beta\left(I-R^{2 N}\right)(I+R) R^{N-1}\left(4 R-R^{2}-I\right) \\
& \times\left(4+4\left(\frac{\lambda}{\tau}-\left[\frac{\lambda}{\tau}\right]\right)\right) \tau B_{h}^{x} f_{[\lambda / \tau]+1}^{h}(x) \\
& +\beta\left(I-R^{2 N}\right)(I+R) R^{N-2}\left(4 R-R^{2}-I\right) \\
& \times\left[2 R\left(R+2\left(\frac{\lambda}{\tau}-\left[\frac{\lambda}{\tau}\right]\right)\right)\right. \\
& +R^{[\lambda / \tau]-1}\left(I-3 R+2\left(\frac{\lambda}{\tau}-\left[\frac{\lambda}{\tau}\right]\right)(I-R)\right)+R^{N-[\lambda / \tau]-2} \\
& \left.\times\left(3 I-R+2\left(\frac{\lambda}{\tau}-\left[\frac{\lambda}{\tau}\right]\right)(I-R)\right)\right] f_{[\lambda / \tau]}^{h}(x) \\
& \times\left[(I+R)\left(I-R^{2 N}\right)\right. \\
& \times\left[( I - 3 R ) \left(3 R-I-R^{2 N-5}(I-R)\right.\right. \\
& \left.\times\left(R^{2}-R^{2 N}(I+R)\left(R^{2}-4 R+I\right)\right)\right) \\
& -(3 I-R) R^{N-1}\left(R^{2}-4 R+I\right)-\beta\left(4 R-I-R^{2}\right) \\
& \times\left(\left(3 I-R+2\left(\frac{\lambda}{\tau}-\left[\frac{\lambda}{\tau}\right]\right)(I-R)\right) R^{N-[\lambda / \tau]-3}\right. \\
& -\left(I-3 R+2\left(\frac{\lambda}{\tau}-\left[\frac{\lambda}{\tau}\right]\right)(I-R)\right) \\
& \left.\left.\left.\times R^{N+[\lambda / \tau]-3}\right)\right]\right] f_{1}^{h}(x) \\
& +(I+R)\left(4 R-I-R^{2}\right)\left(I-R^{2 N}\right) R^{N-2} \\
& \left.\times\left(R-3 I-R^{N-2}(I-3 R)\left(I+R^{N}(I-R)\right)\right) f_{N-1}^{h}(x)\right\} \\
& -D_{\tau}\left(I-R^{2 N}\right)(I-R)^{-1}(I+R) R^{N-2}\left(4 R-I-R^{2}\right) 2 \tau A_{h}^{x} \psi^{h}(x) \\
& +D_{\tau}\left(I-R^{2 N}\right)(I-R)^{-1}
\end{aligned}
$$


14

Abstract and Applied Analysis

$$
\begin{aligned}
& \times\left[3 I-R-R^{2 N-2}(I-3 R)\right. \\
& -\beta\left[R^{N-[\lambda / \tau]}\left(3 I-R+2\left(\frac{\lambda}{\tau}-\left[\frac{\lambda}{\tau}\right]\right)(I-R)\right)\right. \\
& \left.\left.-R^{N+[\lambda / \tau]-1}\left(I-3 R+2\left(\frac{\lambda}{\tau}-\left[\frac{\lambda}{\tau}\right]\right)(I-R)\right)\right]\right] 2 \tau A_{h}^{x} \varphi^{h}(x), \\
& A_{h}^{x} R u_{N}^{h}(x)-f_{N-1}^{h}(x) \\
& =D_{\tau}\left(I+\tau B_{h}^{x}\right)\left(2 I+\tau B_{h}^{x}\right)^{-1} \\
& \times\left\{\left(I-R^{2 N}\right)\right. \\
& \times\left[(I+R)\left(4 R-I-R^{2}\right)(R-3 I)\right. \\
& +\beta\left[(R-3 I)\left(3 I-R+2\left(\frac{\lambda}{\tau}-\left[\frac{\lambda}{\tau}\right]\right)(I-R)\right)\right. \\
& \times R^{N-[\lambda / \tau]+1}-(3 R-I) \\
& \left.\left.\times\left(I-3 R+2\left(\frac{\lambda}{\tau}-\left[\frac{\lambda}{\tau}\right]\right)(I-R)\right) R^{N+[\lambda / \tau]+1}\right]\right] \\
& \times\left(\sum_{i=1}^{N-2} B_{h}^{x} \tau R^{N-i}\left(f_{i}^{h}(x)-f_{N-1}^{h}(x)\right)-\sum_{i=1}^{N-2} B_{h}^{x} \tau R^{N+i}\left(f_{i}^{h}(x)-f_{1}^{h}(x)\right)\right) \\
& +(I+R)\left(I-R^{2 N}\right)\left(4 R-I-R^{2}\right) \\
& \times\left[(I+R) R^{N-2}\left(-4 R+I+R^{2}\right)\right. \\
& -\beta\left[R^{[\lambda / \tau]-1}\left(I-3 R+2\left(\frac{\lambda}{\tau}-\left[\frac{\lambda}{\tau}\right]\right)(I-R)\right)\right. \\
& \left.\left.-R^{2 N-[\lambda / \tau]-1}\left(3 I-R+2\left(\frac{\lambda}{\tau}-\left[\frac{\lambda}{\tau}\right]\right)(I-R)\right)\right]\right] \\
& \times \sum_{i=2}^{N-1} B_{h}^{x} \tau R^{i}\left(f_{i}^{h}(x)-f_{1}^{h}(x)\right) \\
& +\beta R\left(R-3 I+R^{2 N-2}(I-3 R)\right)\left(I-R^{2 N}\right) \\
& \times\left[\left(I-3 R+2\left(\frac{\lambda}{\tau}-\left[\frac{\lambda}{\tau}\right]\right)(I-R)\right)\right. \\
& \times \sum_{i=1}^{[\lambda / \tau]-1} B_{h}^{x} \tau R^{[\lambda / \tau]-i}\left(f_{i}^{h}(x)-f_{[\lambda / \tau]}^{h}(x)\right)
\end{aligned}
$$


Abstract and Applied Analysis

$$
\begin{aligned}
& +\left(3 I-R+2\left(\frac{\lambda}{\tau}-\left[\frac{\lambda}{\tau}\right]\right)(I-R)\right) \\
& \times \sum_{i=[\lambda / \tau]+2}^{N-1} B_{h}^{x} \tau R^{[\lambda / \tau]+i}\left(f_{i}^{h}(x)-f_{1}^{h}(x)\right) \\
& +\left(I-3 R+2\left(\frac{\lambda}{\tau}-\left[\frac{\lambda}{\tau}\right]\right)(I-R)\right) \\
& \left.\times \sum_{i=1}^{N-1} B_{h}^{x} \tau R^{[\lambda / \tau]+i}\left(f_{i}^{h}(x)-f_{1}^{h}(x)\right)\right] \\
& +\beta R^{2}\left(R-3 I+R^{2 N-2}(I-3 R)\right)\left(I-R^{2 N}\right)\left(4+4\left(\frac{\lambda}{\tau}-\left[\frac{\lambda}{\tau}\right]\right)\right) \\
& \times f_{[\lambda / \tau]-1}^{h}(x)-\beta R\left(I-R^{2 N}\right) \\
& \times\left[\left(I-R+R^{N-[\lambda / \tau]}\right)\left(3 I-R+2\left(\frac{\lambda}{\tau}-\left[\frac{\lambda}{\tau}\right]\right)(I-R)\right)\right. \\
& \left.+R^{[\lambda / \tau]-1}\left(I-3 R+2\left(\frac{\lambda}{\tau}-\left[\frac{\lambda}{\tau}\right]\right)(I-R)\right)\right] \\
& \times f_{[\lambda / \tau]}^{h}(x)+\left(I-R^{2 N}\right) \\
& \times\left[(I+R)\left(4 R-R^{2}-I\right) R^{N+1}\right. \\
& \times(R-3 I)\left(I-R^{N-2}\right) \\
& +\beta\left[\left(3 I-R+2\left(\frac{\lambda}{\tau}-\left[\frac{\lambda}{\tau}\right]\right)(I-R)\right)\right. \\
& \times R^{2 N-[\lambda / \tau]}\left(R^{2}(3 I-R)+R^{N-2}\left(R^{3}-4 R^{2}+I\right)\right) \\
& -\left(I-3 R+2\left(\frac{\lambda}{\tau}-\left[\frac{\lambda}{\tau}\right]\right)(I-R)\right) R^{[\lambda / \tau]} \\
& \left.\left.\times\left(R^{2}(3 I-R)+(I-3 R) R^{N-3}\left(R+R^{N}(I-R)\right)\right)\right]\right] \\
& \times f_{1}^{h}(x)+\left(I-R^{2 N}\right) \\
& \times\left[(I-3 R)\left(4 R-R^{2}-I\right)\left(3 I-R+R^{2 N-2}\right)\right. \\
& -\beta\left[\left(3 I-R+2\left(\frac{\lambda}{\tau}-\left[\frac{\lambda}{\tau}\right]\right)(I-R)\right) R^{N-[\lambda / \tau]-1}(3 I-R)\right. \\
& \times\left(R+I+R^{N}\left(I-R+R^{N-2}(I-R)+R^{3 N-2}(2 I-R)\right)\right) \\
& -\left(I-3 R+2\left(\frac{\lambda}{\tau}-\left[\frac{\lambda}{\tau}\right]\right)(I-R)\right) R^{N+[\lambda / \tau]-1}(3 R-I)
\end{aligned}
$$




$$
\begin{gathered}
\times\left(I-R-R^{2 N-3}\right. \\
\left.\left.\left.\times\left(1+R-R^{N}\left(3 I-R^{2}\right)+R^{2 N}\left(R^{3}-4 R^{2}+I\right)\right)\right)\right]\right] \\
\left.\times f_{N-1}^{h}(x)\right\} \\
+D_{\tau}\left(I-R^{2 N}\right)(I-R)^{-1} R\left(R-3 I+R^{2 N-2}(I-3 R)\right) 2 \tau A_{h}^{x} \varphi^{h}(x) \\
-D_{\tau}\left(I-R^{2 N}\right)(I-R)^{-1} R \\
\times\left[R^{N-2}(I+R)\left(R^{2}-4 R+I\right)\right. \\
\quad-\beta\left[R^{[\lambda / \tau]-1}\left(I-3 R+2\left(\frac{\lambda}{\tau}-\left[\frac{\lambda}{\tau}\right]\right)(I-R)\right)\right. \\
\left.\left.\quad-R^{2 N-[\lambda / \tau]-1}\left(3 I-R+2\left(\frac{\lambda}{\tau}-\left[\frac{\lambda}{\tau}\right]\right)(I-R)\right)\right]\right] 2 \tau A_{h}^{x} \psi^{h}(x)
\end{gathered}
$$

for (2.8), the symmetry properties of the difference operator $A_{h}^{x}$ defined by the formula (2.5) and on Theorem 2.3 on well-posedness of the elliptic difference problem.

\section{Numerical Analysis}

We have not been able to obtain a sharp estimate for the constants figuring in the stability inequality. Therefore, we will give the following results of numerical experiments of the Bitsadze-Samarskii-Dirichlet problem:

$$
\begin{gathered}
-\frac{\partial^{2} u(t, x)}{\partial t^{2}}-\frac{\partial^{2} u(t, x)}{\partial x^{2}}+u=f(t, x), \quad 0<t<1,0<x<1, \\
f(t, x)=\exp (-\pi t) \sin (\pi x), \quad 0<t<1,0<x<1, \\
u_{t}(0, x)=-\pi \sin (\pi x), \quad 0 \leq x \leq 1, \\
u_{t}(1, x)=u_{t}\left(\frac{1}{2}, x\right)+\pi \sin (\pi x)\left(\exp \left(-\frac{\pi}{2}\right)-\exp (-\pi)\right), \quad 0 \leq x \leq 1, \\
u(t, 0)=u(t, 1)=0, \quad 0 \leq t \leq 1
\end{gathered}
$$

for the two-dimensional elliptic equation. 
The exact solution of this problem is

$$
u(t, x)=\exp (-\pi t) \sin (\pi x)
$$

For the approximate solution of problem (3.1), we consider the set $[0,1]_{\tau} \times[0,1]_{h}$ of a family of grid points depending on small parameters $\tau$ and $h$ as follows:

$$
\begin{gathered}
{[0,1]_{\tau} \times[0,1]_{h}=\left\{\left(t_{k}, x_{n}\right): t_{k}=k \tau, 0 \leq k \leq N, N \tau=1,\right.} \\
\left.x_{n}=n h, 0 \leq n \leq M, M h=1\right\} .
\end{gathered}
$$

Applying (2.7), we present the following first-order of accuracy difference scheme for the approximate solution of problem (3.1):

$$
\begin{aligned}
& -\frac{u_{k+1}^{n}-2 u_{k}^{n}+u_{k-1}^{n}}{\tau^{2}}-\frac{u_{k}^{n+1}-2 u_{k}^{n}+u_{k}^{n-1}}{h^{2}}+u_{k}^{n}=\exp \left(-\pi t_{k}\right) \sin \left(\pi x_{n}\right), \quad 1 \leq k \leq N-1, \\
& 1 \leq n \leq M-1, \\
& \frac{u_{1}^{n}-u_{0}^{n}}{\tau}=-\pi \sin \left(\pi x_{n}\right), \quad 0 \leq n \leq M, \\
& \frac{u_{N}^{n}-u_{N-1}^{n}}{\tau}=\beta \frac{u_{N / 2}^{n}-u_{N / 2-1}^{n}}{\tau}+\pi \sin \left(\pi x_{n}\right) \\
& \times\left(\exp \left(-\frac{\pi}{2}\right)-\exp (-\pi)\right), \quad 0 \leq n \leq M, \\
& u_{k}^{0}=u_{k}^{M}=0, \quad 0 \leq k \leq N \text {. }
\end{aligned}
$$

We have $(N+1) \times(M+1)$ system of linear equations in (3.4) and we will write them in the following matrix form:

$$
\begin{gathered}
A u_{n+1}+B u_{n}+C u_{n-1}=D \varphi_{n}, \quad 1 \leq n \leq M-1, \\
u_{0}=u_{M}=\widetilde{0} .
\end{gathered}
$$


Here,

$$
\begin{aligned}
& A=\left[\begin{array}{cccccccccc}
0 & 0 & 0 & \cdot & 0 & 0 & \cdot & 0 & 0 & 0 \\
0 & a & 0 & \cdot & 0 & 0 & \cdot & 0 & 0 & 0 \\
0 & 0 & a & \cdot & 0 & 0 & \cdot & 0 & 0 & 0 \\
\cdot & \cdot & \cdot & \cdot & \cdot & \cdot & \cdot & \cdot & \cdot & \cdot \\
0 & 0 & 0 & \cdot & 0 & 0 & \cdot & a & 0 & 0 \\
0 & 0 & 0 & \cdot & 0 & 0 & \cdot & 0 & a & 0 \\
0 & 0 & 0 & \cdot & 0 & 0 & \cdot & 0 & 0 & 0
\end{array}\right]_{(N+1) \times(N+1)} \\
& B=\left[\begin{array}{cccccccccc}
-1 & 1 & 0 & \cdot & 0 & 0 & \cdot & 0 & 0 & 0 \\
c & b & c & \cdot & 0 & 0 & \cdot & 0 & 0 & 0 \\
0 & c & b & \cdot & 0 & 0 & \cdot & 0 & 0 & 0 \\
\cdot & \cdot & \cdot & \cdot & \cdot & \cdot & \cdot & \cdot & \cdot & \cdot \\
0 & 0 & 0 & \cdot & 0 & 0 & \cdot & b & c & 0 \\
0 & 0 & 0 & \cdot & 0 & 0 & \cdot & c & b & c \\
0 & 0 & 0 & \cdot & 1 & -1 & \cdot & 0 & -1 & 1
\end{array}\right]_{(N+1) \times(N+1)} \\
& C=A, \quad D=[I]_{(N+1) \times(N+1)}, \quad u_{s}=\left[\begin{array}{c}
u_{s}^{0} \\
u_{s}^{1} \\
\cdot \\
u_{s}^{N-1} \\
u_{s}^{N}
\end{array}\right]_{(N+1) \times 1},
\end{aligned}
$$

where $s=n-1, n, n+1$ and

$$
\begin{gathered}
\varphi_{n}=\left[\begin{array}{c}
\varphi_{n}^{0} \\
\varphi_{n}^{1} \\
\dot{\varphi^{N}-1} \\
\varphi_{n}^{N} \\
\varphi_{n}^{N}
\end{array}\right]_{(N+1) \times 1}, \quad c=\frac{1}{\tau^{2}} \\
\varphi_{n}^{k}=\left\{\begin{array}{cl}
-\frac{1}{h^{2}}, \quad b=-\frac{2}{\tau^{2}}-\frac{2}{h^{2}}-1, \quad & 1 \leq k \leq N-1, \\
-\tau \pi \sin \left(\pi x_{n}\right), & k=N .
\end{array}\right.
\end{gathered}
$$

We seek a solution of the matrix equation in following form:

$$
\begin{gathered}
u_{n}=\alpha_{n+1} u_{n+1}+\beta_{n+1}, \quad n=M-1, \ldots, 1, \\
u_{M}=\widetilde{0}
\end{gathered}
$$


where $\alpha_{n}(n=1, \ldots, M)$ are $(N+1) \times(N+1)$ square matrices and $\beta_{n}(n=1, \ldots, M)$ are $(N+1) \times 1$ column matrices defined by (see, [17]) as follows:

$$
\begin{aligned}
& \alpha_{n+1}=-\left(B+C \alpha_{n}\right)^{-1} A, \\
& \beta_{n+1}=\left(B+C \alpha_{n}\right)^{-1}\left(D \varphi_{n}-C \beta_{n}\right), \quad n=1, \ldots, M-1,
\end{aligned}
$$

where

$$
\alpha_{1}=[0]_{(N+1) \times(N+1)}, \quad \beta_{1}=[0]_{(N+1) \times 1} .
$$

Now, applying (2.8) for $N$ even number, we can present the following second-order of accuracy difference scheme:

$$
\begin{array}{cc}
-\frac{u_{k+1}^{n}-2 u_{k}^{n}+u_{k-1}^{n}}{\tau^{2}}-\frac{u_{k}^{n+1}-2 u_{k}^{n}+u_{k}^{n-1}}{h^{2}}+u_{k}^{n}=-\exp \left(-\pi t_{k}\right) \sin \left(\pi x_{n}\right), & 1 \leq k \leq N-1, \\
\frac{-3 u_{0}^{n}+4 u_{1}^{n}-u_{2}^{n}}{2 \tau}=-\pi \sin \left(\pi x_{n}\right), \quad 0 \leq n \leq M, & 1 \leq h \leq M-1, \\
\frac{u_{N-2}^{n}(x)-4 u_{N-1}^{n}(x)+3 u_{N}^{n}(x)}{2 \tau}=\frac{u_{N / 2-2}^{n}-4 u_{N / 2-1}^{n}+3 u_{N / 2}^{n}}{2 \tau} & \\
+\pi \sin \left(\pi x_{n}\right)\left(\exp \left(-\frac{\pi}{2}\right)-\exp (-\pi)\right), \quad 0 \leq n \leq M, & \\
u_{k}^{0}=u_{k}^{M}=0, \quad 0 \leq k \leq N &
\end{array}
$$

for the approximate solution of problem (3.1).

So, again we have $(N+1) \times(M+1)$ system of linear equations in (3.11) and we will write them in the following matrix form:

$$
\begin{aligned}
A u_{n+1}+B u_{n}+C u_{n-1} & =D \varphi_{n}, \quad 1 \leq n \leq M-1, \\
u_{0} & =u_{M}=\tilde{0}
\end{aligned}
$$


where

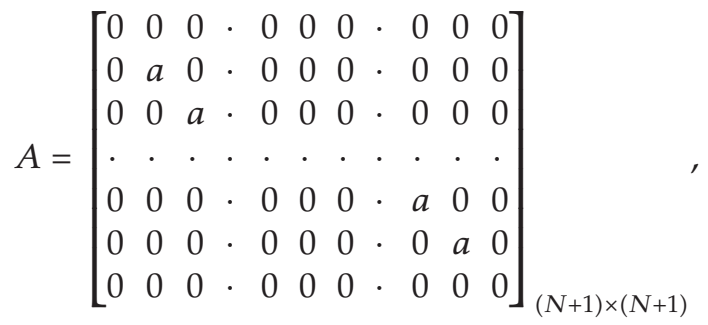

$$
\begin{aligned}
& B=\left[\begin{array}{ccccccccccc}
-3 & 4 & -1 & \cdot & 0 & 0 & 0 & \cdot & 0 & 0 & 0 \\
b & c & b & \cdot & 0 & 0 & 0 & \cdot & 0 & 0 & 0 \\
0 & b & c & \cdot & 0 & 0 & 0 & \cdot & 0 & 0 & 0 \\
\cdot & \cdot & \cdot & \cdot & \cdot & \cdot & \cdot & \cdot & \cdot & \cdot & \cdot \\
0 & 0 & 0 & \cdot & 0 & 0 & 0 & \cdot & c & b & 0 \\
0 & 0 & 0 & \cdot & 0 & 0 & 0 & \cdot & b & c & b \\
0 & 0 & 0 & \cdot & -1 & 4 & -3 & \cdot & 1 & -4 & 3
\end{array}\right]_{(N+1) \times(N+1)} \\
& C=A, \quad D=[I]_{(N+1) \times(N+1)}, \\
& u_{s}=\left[\begin{array}{c}
u_{s}^{0} \\
u_{s}^{1} \\
\cdot \\
u_{s}^{N-1} \\
u_{s}^{N}
\end{array}\right]_{(N+1) \times 1},
\end{aligned}
$$

where $s=n-1, n, n+1$ and

$$
\varphi_{n}^{k}=\left[\begin{array}{c}
\varphi_{n}^{0} \\
\varphi_{n}^{1} \\
\cdot \\
\varphi_{n}^{N-1} \\
\varphi_{n}^{N}
\end{array}\right]_{(N+1) \times 1} .
$$

Here,

$$
\begin{gathered}
a=\frac{1}{h^{2}}, \quad b=\frac{1}{\tau^{2}}, \quad c=-\frac{2}{h^{2}}-\frac{2}{\tau^{2}}-1, \\
\varphi_{n}^{k}= \begin{cases}-2 \tau \pi \sin \left(\pi x_{n}\right), & k=0, \\
-\exp \left(-\pi t_{k}\right) \sin \left(\pi x_{n}\right), & 1 \leq k \leq N-1, \\
2 \tau \pi \sin \left(\pi x_{n}\right)\left(\exp \left(-\frac{\pi}{2}\right)-\exp (-\pi)\right), & k=N .\end{cases}
\end{gathered}
$$

So, we have the second-order difference equation with respect to $n$ with matrix coefficients. To solve this difference equation, we use the same algorithm (3.8) and (3.9).

Now, we will give the results of the numerical experiments. 
Table 1: Comparison of the errors of difference schemes.

\begin{tabular}{lccc}
\hline Difference schemes & $N=M=20$ & $N=M=40$ & $N=M=60$ \\
\hline First-order difference scheme (2.7) & 0.05384197882300 & 0.02631633782987 & 0.01740639813932 \\
Second-order difference scheme (2.8) & 0.00631619894867 & 0.001644554775890 & $7.4144589892985 \mathrm{e}-004$ \\
\hline
\end{tabular}

The errors in numerical solutions are computed by

$$
E_{M}^{N}=\max _{1 \leq k \leq N-1}\left(\sum_{n=1}^{M-1}\left|u\left(t_{k}, x_{n}\right)-u_{n}^{k}\right|^{2} h\right)^{1 / 2}
$$

for different values of $M$ and $N$, where $u\left(t_{k}, x_{n}\right)$ represents the exact solution and $u_{n}^{k}$ represents the numerical solution at $\left(t_{k}, x_{n}\right)$. The results are shown in Table 1 for $N=M=20$, $N=M=40$, and $N=M=60$.

Thus, second-order of accuracy difference scheme is more accurate compared with the first-order of accuracy difference scheme.

\section{Conclusion}

The first and second-orders of accuracy difference schemes for approximate solutions of the Bitsadze-Samarskii-Dirichlet type nonlocal boundary value problem for the multidimensional elliptic partial differential equation are presented. Theorems on the stability, almost coercive stability, and coercive stability estimates for the solution of these difference schemes are established. Numerical experiments are given.

\section{References}

[1] O. A. Ladyzhenskaya and N. N. Ural'tseva, Linear and Quasilinear Equations of Elliptic Type, Nauka, Moscow, Russia, 1973.

[2] M. L. Vishik, A. D. Myshkis, and O. A. Oleinik, "Partial differential equations," in Mathematics in USSR in the Last 40 Years, 1917-1957, vol. 1, pp. 563-599, Fizmatgiz, Moscow, Russia, 1959.

[3] S. G. Krein, Linear Differential Equations in Banach Space, Nauka, Moscow, Russia, 1966.

[4] V. L. Gorbachuk and M. L. Gorbachuk, Boundary Value Problems for Differential-Operator Equations, Naukova Dumka, Kiev, Russia, 1984.

[5] G. Berikelashvili, "On a nonlocal boundary-value problem for two-dimensional elliptic equation," Computational Methods in Applied Mathematics, vol. 3, no. 1, pp. 35-44, 2003.

[6] F. Criado-Aldeanueva, F. Criado, N. Odishelidze, and J. M. Sanchez, "On a control problem governed by a linear partial differential equation with a smooth functional," Optimal Control Applications $\mathcal{E}$ Methods, vol. 31, no. 6, pp. 497-503, 2010.

[7] A. Ashyralyev, "Nonlocal boundary-value problems for elliptic equations: well-posedness in Bochner spaces," in Proceedings of the ICMS International Conference on Mathematical Science, vol. 1309 of AIP Conference Proceedings, pp. 66-84, Bolu, Turkey, November 2010.

[8] A. Ashyralyev, "On well-posedness of the nonlocal boundary value problems for elliptic equations," Numerical Functional Analysis and Optimization, vol. 24, no. 1-2, pp. 1-15, 2003.

[9] I. A. Gurbanov and A. A. Dosiev, "On the numerical solution of nonlocal boundary problems for quasilinear elliptic equations," in Approximate Methods for Operator Equations, pp. 64-74, Baku State University, Baku, Azerbaijan, 1984.

[10] A. A. Samarskii and E. S. Nikolaev, Numerical Methods for Grid Equations, 2 Iterative Methods, Birkhäuser, Basel, Switzerland, 1989. 
[11] A. Ashyralyev, C. Cuevas, and S. Piskarev, "On well-posedness of difference schemes for abstract elliptic problems in $\mathrm{L}_{p}([0,1], E)$ spaces," Numerical Functional Analysis and Optimization, vol. 29, no. 1-2, pp. 43-65, 2008.

[12] A. V. Bitsadze and A. A. Samarskii, "On some simplest generalizations of linear elliptic problems," Doklady Akademii Nauk SSSR, vol. 185, pp. 739-740, 1969.

[13] A. Ashyralyev and E. Ozturk, "Numerical solutions of Bitsadze-Samarskii problem for elliptic equations," in Further Progress in Analysis: Proceedings of the 6th International ISAAC Congress Ankara, Turkey 13-18 August 2007, pp. 698-707, World Scientific, 2009.

[14] A. P. Soldatov, "A problem of Bitsadze-Samarskii type for second-order elliptic systems on the plane," Russian in Doklady Akademii Nauk, vol. 410, no. 5, pp. 607-611, 2006.

[15] D. G. Gordeziani, "On a method of resolution of Bitsadze-Samarskii boundary value problem," Abstracts of Reports of Institute of Applied Mathematics, vol. 2, pp. 38-40, 1970.

[16] D. V. Kapanadze, "On the Bitsadze-Samarskii nonlocal boundary value problem," Differential Equations, vol. 23, no. 3, pp. 543-545, 1987.

[17] A. Ashyralyev, "A note on the Bitsadze-Samarskii type nonlocal boundary value problem in a Banach space," Journal of Mathematical Analysis and Applications, vol. 344, no. 1, pp. 557-573, 2008.

[18] R. P. Agarwal and V. B. Shakhmurov, "Multipoint problems for degenerate abstract differential equations," Acta Mathematica Hungarica, vol. 123, no. 1-2, pp. 65-89, 2009.

[19] V.Shakhmurov and R. Shahmurov, "Maximal B-regular integro-differential equation," Chinese Annals of Mathematics B, vol. 30, no. 1, pp. 39-50, 2009.

[20] D. Orlovsky and S. Piskarev, "On approximation of inverse problems for abstract elliptic problems," Journal of Inverse and Ill-Posed Problems, vol. 17, no. 8, pp. 765-782, 2009.

[21] P. E. Sobolevskii, Difference Methods for the Approximate Solution of Differential Equations, Voronezh State University, Voronezh, Russia, 1975. 


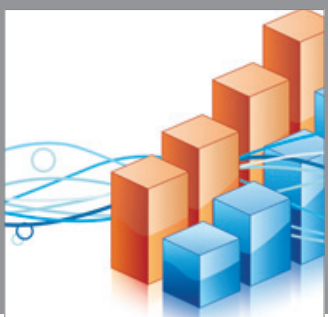

Advances in

Operations Research

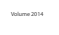

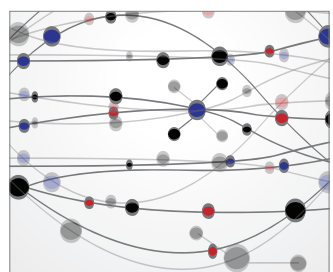

\section{The Scientific} World Journal
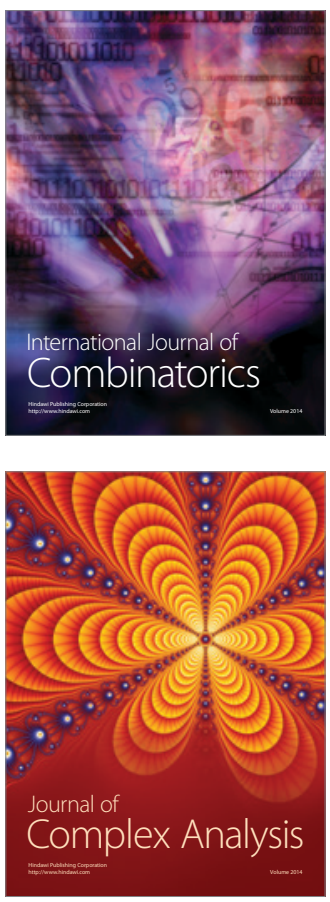

International Journal of

Mathematics and

Mathematical

Sciences
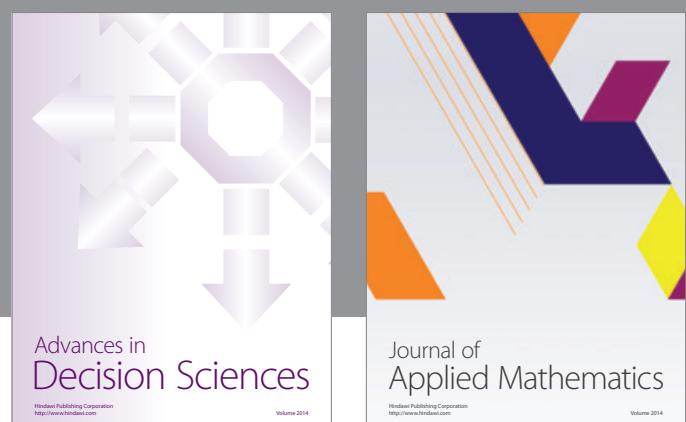

Journal of

Applied Mathematics
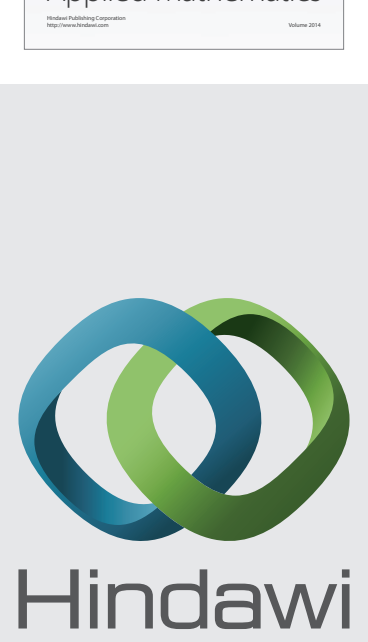

Submit your manuscripts at http://www.hindawi.com
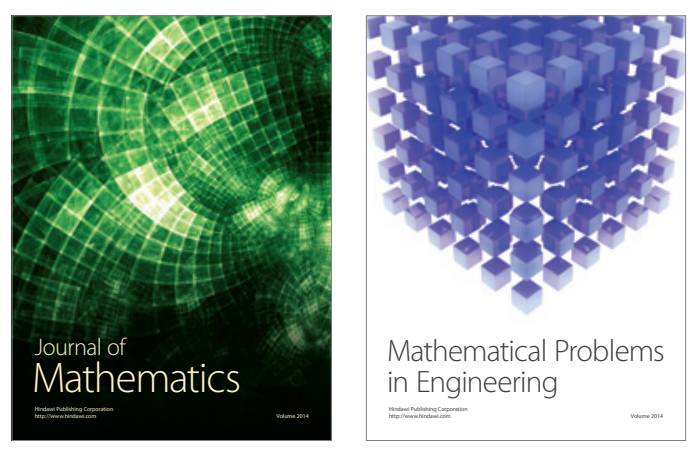

Mathematical Problems in Engineering
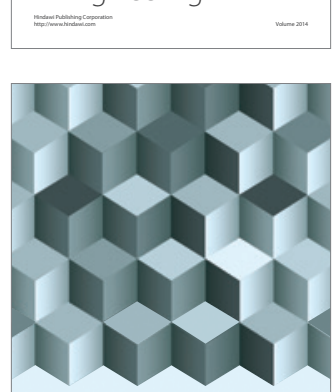

Journal of

Function Spaces
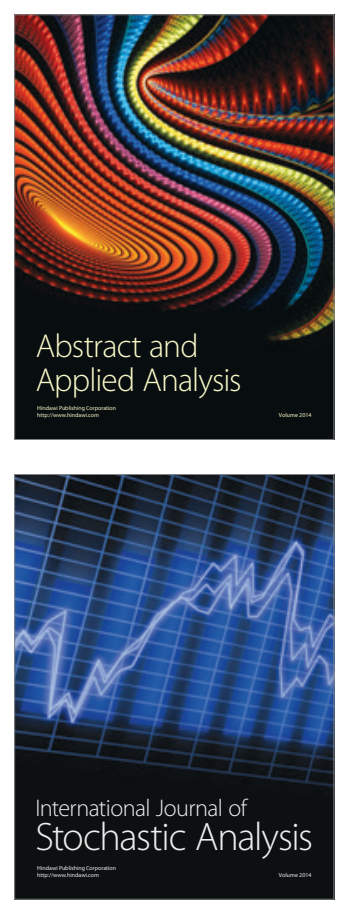

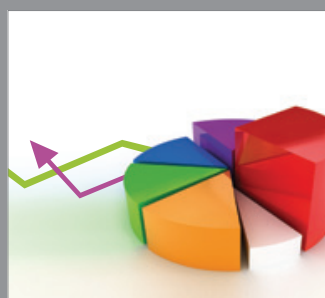

ournal of

Probability and Statistics

Promensencen
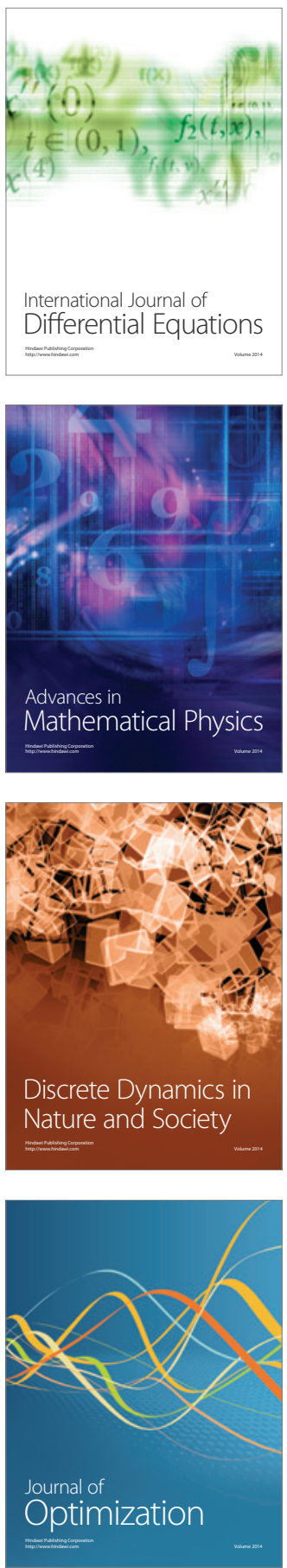